\title{
La fondation de l'Empire ottoman chez Kemal Tahir et Tarik Bugra entre mythe et réalité historique
}

The foundation of the Ottoman Empire in the works of Kemal Tahir and Tarik Bugrra. Between the myth and the historical reality

\section{Valérie Gay}

\section{(2) OpenEdition}

\section{Journals}

Édition électronique

URL : https://journals.openedition.org/ceb/1578

DOI : 10.4000/ceb.1578

ISSN : 2261-4184

Éditeur

INALCO

Édition imprimée

Date de publication : 1 décembre 2008

Pagination : 369-398

ISBN : 978-2-85831-173-6

ISSN : 0290-7402

Référence électronique

Valérie Gay, «La fondation de l'Empire ottoman chez Kemal Tahir et Tarik Bugra entre mythe et réalité historique », Cahiers balkaniques [En ligne], 36-37 | 2008, mis en ligne le 20 mai 2012, consulté le 06 juillet 2021. URL : http://journals.openedition.org/ceb/1578; DOI : https://doi.org/10.4000/ceb.1578

Ce document a été généré automatiquement le 6 juillet 2021

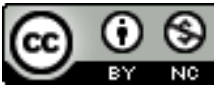

Cahiers balkaniques est mis à disposition selon les termes de la Licence Creative Commons Attribution - Pas d'Utilisation Commerciale 4.0 International. 


\section{La fondation de l'Empire ottoman chez Kemal Tahir et Tarik Bugra entre mythe et réalité historique}

The foundation of the Ottoman Empire in the works of Kemal Tahir and Tarik Bugrra. Between the myth and the historical reality

Valérie Gay

1 La naissance de l'État ottoman est devenue un vaste sujet de débat académique qui s'enflamme de temps en temps. Les origines des Ottomans et leur histoire au moins jusqu'au $\mathrm{XIV}^{\mathrm{e}}$ siècle ont longtemps été mal connues. La rareté des sources et les divergences entre elles, des documents historiques reconstruits a posteriori et fondés sur des légendes ont contribué au fait que les historiens, jusqu'à une période récente, n'ont utilisé que des sources partiales, hostiles ou visant à magnifier la dynastie ottomane. Des documents nouveaux, pour la plupart provenant des archives ottomanes et européennes, et une analyse critique des chroniques ont permis de mieux cerner cette période des débuts de l'État ottoman, où des points de contestation, comme la datation et les facteurs ayant contribué à l'émergence de cet Empire, contribuent à alimenter les débats.

2 En choisissant de raconter cette période obscure de l'histoire ottomane dans leurs romans Devlet Ana (La mère Patrie) et Osmancık (Le petit Osman), Kemal Tahir et Tarık Buğra ont suscité à la fois un vif enthousiasme et de violentes critiques. Autant de réactions qui s'inscrivent dans le contexte idéologique de la Turquie républicaine, où l'histoire semble être le domaine privilégié du dogmatisme. Pour comprendre les débats occasionnés par ces œuvres, qui posent la question de la vérité historique et de sa représentation littéraire, il est nécessaire d'étudier le sujet à la lumière de trois aspects imbriqués: le débat historiographique, la manière dont il se reflète dans le roman turc et le contexte politique et culturel dans lequel ont vécu les deux auteurs.

Dans une première partie consacrée à la présentation de Kemal Tahir et Tarık Buğra, nous reviendrons sur quelques caractéristiques de la littérature turque pour montrer la 
place particulière qu'occupent ces deux auteurs, distincts au départ par la dimension du marxisme et de l'Islam, mais qui se rejoignent dans la même opposition aux tabous historiques officialisés par le régime républicain. Dans une seconde partie, à travers l'analyse des romans Devlet Ana et Osmanck, nous verrons que si leur choix d'interprétation des sources historiques diffère au départ, les particularités de cette période historique ainsi que le but présidant à l'écriture des romans les mènent à recourir à des modèles formels relevant du mythe et que leur vision enthousiaste pour cette époque les conduit à des conclusions similaires. Dans une troisième partie, nous montrerons que finalement, le marxiste Kemal Tahir et le défenseur de l'Islam Tarık Buğra, se retrouvent dans une même défense des valeurs ottomanes et de la culture nationale contre les courants d'occidentalisation. Ainsi, à travers les distorsions qui s'opèrent entre leur appel à l'objectivité historique et leur revendication d'une liberté d'interprétation littéraire, il apparaît clairement que si les termes de réalité et de mythe créent autant de polémiques (outrepassant toujours le fait littéraire), c'est qu'en amont et en aval de l'écriture, ce sont bien des critères concernant l'élaboration de l'identité et d'une mythologie nationales dont il s'agit. Dans le contexte d'une littérature où la dimension sociopolitique est omniprésente, la question au cœur des débats est finalement celle de la définition de la réalité. Et dans le contexte d'une pensée partisane, cette vérité tend souvent à être envisagée comme un absolu par celui qui l'énonce. Lorsque la pluralité est impossible, c'est rarement le consensus, mais souvent la discorde qui est devient absolue.

\section{Le roman historique par Kemal Tahir et Tarık Buğra : une nouveauté controversée}

\section{La littérature turque à l'aune de l'histoire}

4 Même lorsqu'il ne s'agit pas de roman historique stricto sensu, l'un des traits dominants qui apparaît dans l'ensemble du roman turc, c'est que l'histoire est l'une de ses principales sources d'inspiration. Dans l'un de ses ouvrages, Taner Timur en donne l'explication suivante: "On ne trouve pas dans l'héritage ottoman de tradition de pensée philosophique et sociologique. C'est la raison pour laquelle une certaine vision historique a endossé le rôle que joue habituellement la philosophie dans d'autres cultures. Ce sont les historiens qui orientent la vie intellectuelle en Turquie $»^{1}$. D'autre part, il était nécessaire que la Guerre d'indépendance, devenue une part de la mémoire collective, acquière une dimension historique ainsi que toutes les institutions de l'État moderne issu de la Révolution turque, avec ses références et ses symboles. Cependant, beaucoup d'intellectuels turcs contemporains s'accordent sur le fait que la situation a été très différente en réalité et que les idées républicaines, au lieu de devenir propriété collective, ont le plus souvent été cachées, par Atatürk lui-même, comme s'il s'agissait d'un secret national. À l'aide de quelques retouches à l'époque du multipartisme, on a fait de l'histoire des réformes ottomanes - qui était en fait une histoire de semi colonisation - une histoire de la démocratie turque. Une histoire officielle a donc été instaurée par les forces qui se trouvent au pouvoir en fonction de leurs propres besoins.

Dans la jeune République turque en quête de son identité nationale, le roman s'est généralement accordé à la vision officielle de l'État kémaliste, une vision qui se fonde entièrement sur une rupture avec un Empire ayant duré six siècles. Pour la nouvelle 
idéologie nationale, l'Empire ottoman a symbolisé le passé dont il fallait se débarrasser à tout prix. Dans la ligne des mouvements d'occidentalisation apparus depuis la fin du $\mathrm{XVIII}^{\mathrm{e}}$ siècle, cet Empire a finalement été stigmatisé comme l'obstacle majeur au progrès voulu par les réformistes. L'entreprise de règlement de comptes avec le passé ainsi que la question de l'identité nationale à construire sur les ruines de la structure communautaire ottomane ont orienté les écrivains vers l'exploration de nouvelles voies. C'est ainsi que dans les années proches de la fondation de la République, des sujets comme les raisons de l'effondrement de l'Empire, la Première Guerre mondiale, la période hamidienne, les guerres russo-turques, la défaite balkanique, l'occupation d'Istanbul et les campagnes de combat national entrent dans les domaines d'intérêt des écrivains. Au total, le nombre de romans prenant pour sujet le monde ottoman à d'autres périodes que celles de son effondrement, ou plus exactement, dont l'approche et l'interprétation historiques vont à l'encontre des modèles établis, paraissent très minoritaires ${ }^{2}$.

L'exception la plus célèbre est le roman de Kemal Tahir, Devlet Ana, qui paraît en 1967 et obtient le prix du roman de la Türk Dili Kurumu (Fondation pour la langue turque) en 1968. Suite à une proposition de film historique qui ne verra jamais le jour, Kemal Tahir s'attelle à la rédaction de ce roman qui créera beaucoup de remous. Il raconte la fondation de l'Empire ottoman dans le cadre de la pensée marxiste, qui tendait à dominer sur la scène des idées dans les années 50-60, tout en essayant de définir les particularités de la société turque et de présenter l'âme turque dans son image originelle. Considéré comme une œuvre révolutionnaire par certains intellectuels, qui y trouvaient l'application concrète aux conditions orientales de l'Empire ottoman du concept marxiste de Système de Production de Type Asiatique ${ }^{3}$, cloué au pilori par d'autres, ce roman valut à son auteur d'être affublé des titres de "théoricien », de "fondateur d'école historique », de «tahirisme » ${ }^{4}$ par ses détracteurs. On trouvait qu'il se fourvoyait avec l'idée d'âme turque spécifique, qu'il avait mis sens dessus dessous les réalités historiques et que ce roman était tout simplement illisible.

Le retentissement de Devlet Ana fut tel, qu'il explique en partie la parution tardive du roman de Tarık Buğra : «[...] Malgré tout - voilà, je l'avoue - Osmancık n'a peut-être pas pu être écrit à cause de Devlet Ana. J'avais peur que l'on croie que je l'avais écrit en réaction ${ }^{5}$. » Le déclic se fera après 1981, avec la commande d'un scénario par la TRT (Radio Télévision Turque) sur le thème de la fondation de l'État ottoman, et lorsque le directeur d'édition du journal Tercüman lui propose d'écrire un roman sur le même sujet, Tarık Buğra se lance enfin dans la rédaction de Osmancık qui était en germination depuis plus de 20 ans. À sa parution en 1983, Osmancık est salué la même année par le prix littéraire de la Fondation Nationale de la Culture. Son adaptation deux ans plus tard au Théâtre National d'Ankara provoquera beaucoup de réactions. Comment dans un théâtre fondé par Atatürk pouvait-on parler du Coran et mettre en scène des professions de foi ? Encouragé par le succès de l'adaptation télévisée de Küçük Ağa en 1984, Yücel Çakmaklı réalise une série de 12 épisodes qui seront diffusés sous le titre de Kuruluş (La Fondation) du 10 janvier au 27 mars 1988. Ce sera l'une des plus grosses productions de l'histoire de la télévision turque et surtout l'une des plus critiquées. La difficulté de Çakmaklı à tourner les scènes de guerre et certaines longueurs et erreurs de montage avivèrent encore les critiques.

7 Récompensés en tant qu'œuvres littéraires, c'est sur l'aspect historique que ces deux romans suscitent le plus de critiques. Au-delà de la question de la réussite artistique, 
c'est la manière de traiter l'histoire qui met le feu aux poudres. Et à travers ce qui est souligné comme des erreurs historiques par les critiques, c'est également le but présidant à l'écriture des romans qui pose question. Ainsi, en abordant sous un angle différent des sujets touchant aux mêmes thèmes et périodes historiques, un marxiste et un homme de droite défenseur de la religion ont donné l'impression d'incarner des alternatives complémentaires l'une de l'autre et leurs particularités poussent à la comparaison.

\section{Un climat conflictuel entre droite et gauche}

Que ce soit par leurs œuvres, les interviews, les articles de presse ou les débats auxquels ils participent, Kemal Tahir (1910-1973) et Tarık Buğra (1918-1994) deviennent deux figures importantes de la vie intellectuelle et littéraire dans le contexte très houleux des années 50-60, qui ne fera que s'envenimer jusqu'au troisième coup d'État militaire de 1980. Ils ont eu l'occasion de se côtoyer dans les débats littéraires auxquels ils participent avec les écrivains du réalisme social qui apparaissent avec une nouvelle forme d'expression surtout à partir de 1960.

Kemal Tahir écrit la majeure partie de son œuvre en prison. Ayant travaillé pendant six ans à Bâb-ı Âlî, le quartier de la presse à Istanbul, il est arrêté en 1938 avec Nazım Hikmet et plusieurs civils et militaires, tous inculpés pour leurs idées politiques. Sous la houlette de Nazım Hikmet, dont le rôle sur sa formation d'écrivain est déterminant au cours de ces années de réclusion ${ }^{6}$, Kemal Tahir développe ses idées et son style, et devient rapidement une référence dans les milieux marxistes. La particularité de Kemal Tahir, c'est qu'il ne se contente pas de faire du roman un seul moyen d'expression littéraire, mais le véhicule de connaissances relevant des domaines de l'histoire, de la sociologie et de l'économie, qu'il développe dans de longs dialogues. D'autre part, sa conviction que la société turque est différente de la société occidentale et qu'en raison de cela, elle a besoin d'un roman spécifique et les conceptions théoriques qu'il a développées par la suite sur ce roman, ne peuvent être pensées en dehors de ses thèses. Les idées de Kemal Tahir auront une grande influence, notamment dans le domaine du cinéma où il collabore à plusieurs scénarios.

10 Le parcours plus bohème de Tarık Buğra passe par le Küllük Kahvesi (Le café du cendrier) de Beyazit, un haut lieu du monde littéraire, artistique et scientifique, où il rencontre des personnalités importantes comme Yahya Kemal, Ahmed Hamdi Tanpinar et Fuad Köprülü. Nouvelliste, dramaturge et romancier, Tarık Buğra travaillera également jusqu'à la fin de sa vie comme chroniqueur et critique littéraire. Ses articles, qui traitent généralement des dimensions nationales et traditionnelles, lui valent l'étiquette de "nationaliste de la culture». Son opposition viscérale au marxisme comme à tous les régimes totalitaires et ses positions artistiques le classent comme un nationaliste de droite et un défenseur des valeurs de l'Islam. Pour Buğra, en effet, il est impossible de penser le monde turc en dehors de la religion en tant que composante fondamentale de la culture et de la société. Si de manière générale les qualités d'écriture de Tarik Buğra ne sont pas remises en cause, les débats sur ses œuvres portent sur la place qu'il accorde à l'Islam et sur le fait que les événements qu'il décrit soient appréciés à la lumière de cette idéologie qui le met en porte-à-faux à la fois avec les mouvements de gauche et certains préceptes du kémalisme. 
11 Nés dans un pays encore ottoman, ces deux auteurs ont été les témoins d'un demi-siècle de bouleversements politiques et culturels incessants, dont la réforme linguistique. Pour aborder les œuvres de Kemal Tahir et Tarık Buğra, et surtout analyser correctement leurs propos notamment lorsqu'il s'agit des termes de réalisme et d'objectivité au cœur du débat qui nous intéresse, il est nécessaire de constamment garder à l'esprit le contexte qui était le leur, un contexte du "tout politique » où les étiquettes se collent aussi rapidement que fusent les insultes en brouillant les pistes. Dans le contexte de la guerre froide, la Turquie fait de la Russie et du communisme un seul et même ennemi et se félicite de son entrée à l'OTAN. Avec la constitution de 1961 qui amène un certain assouplissement, les courants de gauche, qui se sont rapidement organisés en associations et divers partis, acquièrent un important poids culturel et se présentent comme les seuls progressistes. Ainsi, dès le début des années 60 , droite et gauche trouvent leur ligne de démarcation définitive et les voies des écrivains et des poètes, qui auparavant pouvaient écrire dans les mêmes revues et se réunir dans les mêmes lieux, se divisent. Le fractionnement du pays en camps idéologiques n'épargne aucun domaine et loin d'être un bastion retranché dans le domaine de l'art, la littérature devient dès lors une importante ligne de front.

\section{Le retour aux sources de l'histoire}

« En Turquie, le roman a changé. [Les œuvres de] Yakup Kadri, Halide Edip aujourd'hui ont beaucoup vieilli. Je ne récuse pas les valeurs de leur époque. Mais ça a vieilli. Kemal Tahir est une nouveauté. Tarlk Buğra est une nouveauté. Même si nous racontons des périodes anciennes. [...] Même si nous nous retournons vers le passé, nous ne sommes pas dans la perspective des romans actuels. Notre vision de l'humain et les valeurs que nous accordons à la société sont très différentes $»^{7}$.

Pour comprendre la nouveauté et la différence dont parle ici Tarık Buğra, rappelons encore une fois que dans les œuvres définies comme "Romans de la Guerre d'Indépendance ", on voit se perpétuer tout au long de la période républicaine depuis les années 1920, le récit d'une expérience vécue d'enthousiasme national et ce qu'on pourrait qualifier de ferveur historique. C'est également à cette période que l'on voit apparaître une analyse sociale de diverses périodes de l'histoire, une réflexion sur les événements historiques et l'émergence d'une certaine critique. À la fin des années 1940, les évolutions qui apparaissent dans les domaines économique, social et politique se répercutent inévitablement sur le roman turc. Ayant adopté les modèles du roman occidental depuis les Tanzimat, celui-ci poursuit dans la voie du réalisme social. Mais cette approche n'est pas suffisante pour Kemal Tahir et Tarık Buğra, qui reprochent à ce type de réalisme d'être avant tout compris comme un effort de description, fondé sur une observation superficielle des faits. Selon eux, un authentique romancier doit s'appuyer sur un système de pensée et être porteur d'une vision du monde, fondée sur des principes et un système de valeurs inscrits dans une tradition, capable d'éclairer la perception des faits et des rapports de l'individu avec la société. Seule une vision du monde enracinée, l'Islam pour l'un ou la dialectique pour l'autre, permet de descendre dans les profondeurs de l'histoire et de l'humain, au-delà des transformations visibles, au-delà de la seule observation et de l'aspect documentaire du roman qu'ils récusent.

13 C'est dans ce sens qu'ils ont convoqué l'histoire, car un écrivain qui négligerait l'histoire ne peut être en mesure de comprendre et d'analyser correctement la situation contemporaine. Plus exactement, ce sont les doutes et les questions du présent qui 
poussent à s'orienter vers le passé, pour en comprendre les clefs et adopter l'attitude convenable. Pour les deux auteurs, la connaissance de son histoire par un individu et une société est l'une des conditions de base de son existence et le fait de rompre le lien à l'histoire, de laisser une société sans histoire, c'est la laisser sans armes et sans défense. Kemal Tahir et Tarık Buğra soutiennent de la même manière que le train de réformes de la période républicaine qui visaient à couper le peuple turc de son histoire et les mesures prises pour éviter tout retour en arrière ont créé beaucoup de dégâts. C'est la raison pour laquelle ils jugent indispensable de comprendre et de commenter d'une manière juste l'histoire ancienne et récente, et de la transmettre aux jeunes générations, pour qui il est urgent de recevoir l'enseignement d'une histoire loin des travestissements et des jugements adoptés comme des vérités absolues. Accordant tous deux une importance fondamentale à l'histoire, ils s'entendent sur le fait que la littérature à thème historique n'est pas l'histoire. Mais l'approche de Kemal Tahir et de Tarık Buğra diffère quelque peu sur la question du rôle de l'art et du romancier.

Pour Kemal Tahir, la mission première du romancier turc est d'éclairer le lecteur. C'est la raison pour laquelle le travail d'un écrivain englobe également celui du sociologue, de l'historien, de l'économiste. Pour Tarık Buğra au contraire, la littérature doit s'en tenir à son propre domaine. La littérature est une humanisation de l'histoire et la seule vérité possible est celle de l'auteur, surtout lorsqu'il s'agit d'apporter un éclairage sur une période perdue dans les abîmes du temps et qui se prête particulièrement à l'interprétation. À propos d'Osmancik il déclare : «La vision historique ou quoi que ce soit ne m'intéresse absolument pas. Ce n'est pas mon affaire. Je ne suis pas parti avec en tête l'idée de défendre ou de dénigrer telle conception ou telle interprétation. J'ai simplement essayé de comprendre et raconter le sujet. Je l'ai raconté tel que je l'ai compris $»^{8}$. Et dans ce sens, il revendique le droit de parler librement de l'Islam, car faire l'impasse sur la religion, qui est une composante fondamentale de la société, et l'exclure pour des motifs idéologiques revient à nier la culture. Cette conviction le fera constamment s'opposer au marxisme, qu'il juge incompatible avec le principe d'indépendance de la littérature, et à tous les régimes totalitaires. Une opposition qui trouvera sa plus forte expression dans des pièces de théâtre comme Ayakta Durmak Istiyorum (Je veux rester debout), qui raconte la résistance des Hongrois contre l'invasion soviétique et Yüzlerce Çiçek Birden Açtı (Soudain s'ouvrirent des centaines de fleurs) qui parle de la Chine maoïste. Avec Küçük Ağa (Le petit agha), qui paraît en 1963, Tarık Buğra acquiert une place spécifique dans le roman turc. En créant le personnage du Hoca d'Istanbul dont le drame est d'être patriote tout en restant fidèle à une tradition de pensée de 600 ans, Tarık Buğra va à l'encontre des clichés décrivant les religieux et les notables comme forcément arriérés, obtus, voire comme des ennemis, et il montre que la victoire du combat national a été possible grâce à la convergence de toutes les composantes d'une société qui formait un tout. Pour l'auteur, le sentiment patriotique, religieux et la conscience nationale étaient mêlés pour la majorité du peuple et ce mouvement qui a pénétré en Anatolie n'était pas pensé en dehors du religieux.

15 Kemal Tahir, quant à lui, pense que la religion et le poids des traditions retiennent le peuple et l'empêchent d'avancer et l'on chercherait en vain, dans la description pessimiste de la réalité rurale dans ses premiers romans, la perspective révolutionnaire présente chez un Yaşar Kemal par exemple. Ainsi, dans la trilogie que constituent Yediçınar Yaylası, Köyün Kamburu et Büyük Mal, qui traitent une période allant de la guerre de Crimée aux dernières années du parti unique dans les régions de Çorum et de 
Çankırı, Kemal Tahir montre que les efforts de centralisation des Tanzimat, la proclamation de la Constitution en 1908 et les réformes kémalistes par la suite n'ont rien changé. Sa défense du principe d'un État fort soutenu par des intellectuels éclairés, capables de mener les réformes dont le peuple a besoin, est en même temps ce qui explique la critique des divers gouvernements qui apparaît dans l'ensemble de ses œuvres, qui couvre une période allant de la fin du XIXe siècle à la fin des années 1950 et reflète tout le déroulement d'une période historique très dense.

La philosophie du matérialisme historique le pousse à analyser non seulement les rouages de la société, mais d'où ils proviennent et la manière dont ils se sont développés. À partir des années 1960-1961, un changement radical s'opère dans la conception de l'auteur qui se met à étudier avec une pensée systématique l'organisation de l'État et la structure socio-économique des sociétés orientales et de l'Empire ottoman. Ne pouvant se satisfaire des explications et des modèles approuvés jusque-là concernant les réalités de l'Empire ottoman et de la Turquie, il étudie toutes les sources turques et étrangères de manière critique, à la lumière de la dialectique. C'est en fonction de cette démarche fondée sur le doute scientifique qu'il réexamine une par une les raisons de la chute de l'Empire et toutes les entreprises de réformes, en soulignant la responsabilité de l'Occident capitaliste et d'une occidentalisation de surface. Kemal Tahir explique ainsi la nécessité de se repencher sur le concept d'ottomanisme, une nécessité qui apparaît pour la première fois face au problème des âyân (notables) qu'il examine dans Köyün Kamburu (Le tors du village) : "À la suite des recherches que j'ai effectuées, je me suis aperçu que les Ottomans ne sont pas les Ottomans que l'on a en mémoire. Ils ont une organisation qui leur est propre. Il faut qu'ils aient des fondements importants pour résister en reculant centimètre par centimètre pendant 300 ans face à une force aussi impitoyable que l'occident. C'est ainsi que le problème des âyân m'a amené à la question des Ottomans. Aujourd'hui je crois que sans comprendre les Ottomans de manière correcte, sans descendre dans cette réalité, nous ne pouvons aborder aucun problème de notre pays. Qu'on les aborde et l'on se trompera'. »

17 L'exigence intellectuelle de ces deux écrivains qui se réclament de systèmes de pensée semblant aux antipodes l'un de l'autre, les a toujours poussés à conspuer le manichéisme simpliste de certaines œuvres et de certains comportements, même s'ils défendaient des idées similaires aux leurs. En cherchant à comprendre et à approfondir l'histoire du pays, en s'efforçant de renouveler le roman, aussi bien au niveau de la forme que de la pensée, Kemal Tahir et Tark Buğra, dont les noms viennent immédiatement à l'esprit quand on parle du roman historique turc, ont produit des œuvres qui les placent en dehors des catégories habituelles du genre. En traitant des thèmes politiques communs comme la Guerre d'indépendance, la montée d'une classe de propriétaires fonciers (ağa) en Anatolie, la critique du Parti unique d'Inönü, ils cherchent à exprimer, à travers leur propre vision du monde, une vérité historique qu'ils jugent déformée et ensevelie sous les credo de l'histoire officielle. À travers Devlet Ana et Osmancik, nous allons voir que ces deux auteurs se rejoignent sur plusieurs points, car ils partent en fait de la même question: Comment un peuple qui a été capable, à partir d'une modeste tribu, de fonder un Empire dont la dimension et la longévité n'ont pas d'équivalent dans le monde, peut-il renier son histoire et se renier lui-même au point de chercher une nouvelle identité dans le miroir que lui tend l'Occident? Leur but essentiel est finalement de réparer l'injustice qu'ils jugent avoir été commise par l'histoire envers les premiers Ottomans. 


\section{Les matériaux de l'histoire et de la fiction}

\section{Deux récits d'une même page d'histoire}

18 tribus turkmènes, qui au XIII ${ }^{e}$ siècle n'étaient encore que des beylik (émirats) des confins dans les frontières byzantines. Ertuğrul et ses proches se trouvent confrontés à deux grands ordres sociaux. D'un côté, l'Empire byzantin ébranlé par les Francs des croisades et les prétentions des gouverneurs locaux chrétiens à élargir leur influence (à instaurer un ordre féodal selon Kemal Tahir). D'un autre côté, l'Empire des Seldjoukides en Anatolie qui vacille sous les attaques des Mongols. D'autre part, les émirats turcs de plus en plus autonomes se battent entre eux pour le trône de Konya. Mais dans ce chaos, c'est du côté d'une modeste tribu turkmène, les Kayl, que se profilent les prémices d'une ère nouvelle.

Devlet Ana débute par un long dialogue entre Mavro, un jeune Grec qui tient le han de son père et Notüs Gladyüs, un chevalier de l'ordre de Saint-Jean, tout frais débarqué en Anatolie pour mettre à exécution ses projets de conquête. Le han, perché sur un rocher qui surplombe une zone de marécages, permet au regard d'embrasser toute la région jusqu'à Bursa, c'est-à-dire la scène de presque tous les événements passés et à venir concernant cette période. Ce dialogue a pour fonction essentielle d'informer le lecteur sur la situation historique, en donnant des informations générales concernant les Turcs de Söğüt et les autres ordres sociaux, de présenter certains personnages et surtout, de poser d'emblée le thème fondamental du livre : l'inconciliable différence entre Orient et Occident. Dès les premières pages, Kemal Tahir essaie de montrer comment le féodalisme occidental essaie d'abattre l'ordre spécifique aux Byzantins et aux Turcs d'Asie Mineure. Ertuğrul Bey est chargé de diriger les confins de Bithynie, dont le sultanat seldjoukide de Konya se sert comme d'un gendarme régional. En interdisant les pillages, Ertuğrul a réussi à maintenir la paix pendant de longues années avec les gouverneurs chrétiens des environs, malgré les excitations à la guerre des tribus frontalières. Mais le contexte général de l'Anatolie, avec l'affluence et le déplacement croissants de populations sous la pression des Mongols, a des répercussions sur la situation intérieure du beylik.

Cette situation est montrée comme apocalyptique dans Devlet Ana. Les Mongols qui dévastent le pays obligent les populations à leur verser de lourds impôts. L'État seldjoukide, en proie à des guerres intestines, entre alors dans une faillite économique qui lui sera fatale. Quant aux terres arides d'Anatolie, elles ne peuvent produire de manière régulière à cause de catastrophes naturelles. Le cours changeant du fleuve Sakarya qui marque la frontière entre Byzance et les émirats turcs, forme des marécages. Les terres sont en friche, le blé est à prix d'or, les troupeaux ont diminué, les échanges sur les marchés sont réduits et le peuple se débat dans une grande pauvreté. Les routes se sont effacées et en raison de l'insécurité des voies, les caravanes ne passent plus. L'Anatolie abonde en effet en brigands, faux abdal et faux derviches. Les vols et les pillages augmentent et le trésor est vide, du fait de la générosité d'Ertuğrul le trésor est vide. De plus, celui-ci est âgé et malade, et son frère Dündar Alp se prépare à la bataille pour la succession contre Osman. C'est à ce moment que le chevalier Notüs Gladyüs passe à l'action. Pensant profiter de l'affaiblissement de 
Byzance pour instaurer un ordre féodal dans les confins, le chevalier comprend qu'il doit d'abord se débarrasser de ce " Turkmène de 90 ans et grabataire » pour réaliser ses projets. Avec l'aide des moines et de certains gouverneurs chrétiens s'appuyant sur un ordre similaire à celui des Francs, il cherche à introduire le désordre dans les tribus frontalières. À côté de Notüs Gladyüs, se trouve un mercenaire des chevaliers de Saint Jean, le guerrier turc Uranha. Sous la conduite du moine Benito, ils entreprennent les actions sanglantes qu'ils ont planifiées. Ils tuent le dresseur de chevaux d'Ertuğrul et sa petite amie grecque Liya, (la sœur de Mavro) et maquillent ce meurtre comme s'il était le fait des voisins grecs de la tribu des Kayı. L'intention est de ruiner l'amitié qui a toujours existé entre le gouverneur chrétien de Karacahisar, Ertuğrul et Osman, et ainsi d'ouvrir le différend entre Grecs et Turcs. Osman ne tombe pas dans le piège, mais il doit mettre un frein au camp de son oncle Dündar, qui sous prétexte de se venger des meurtriers, voit là une occasion de reprendre les pillages. De ce premier fait découle une cascade d'événements qui peu à peu, de pièges en accrochages, vont pousser la tribu d'Osman à l'action et à l'expansion.

21 Tarık Buğra nous présente les faits sous la forme des souvenirs d'Osman. Osman Gazi Han, au seuil de la mort, lutte et demande un délai, le temps nécessaire pour être sûr que son désir de reposer à Bursa va se réaliser. C'est l'heure de l'introspection et des comptes rendus intérieurs. Avec l'annonce de la prise de Bursa par son fils Orhan, se fait la preuve que sa vie a été accomplie et agréée dans sa totalité. Cette dernière lutte intérieure est le noyau à partir duquel s'organisent tous les événements du roman. Le roman comporte un sous-titre qui explicite la démarche de l'auteur : "Cihân devletini kuran irâde, şûur ve karakter » (La volonté, la conscience et le caractère qui fondèrent un État universel». Tarık Buğra s'est penché sur la formation de la personnalité individuelle et politique du personnage d'Osman, et sur les relations réciproques entre l'individualité d'Osman et les faits sociohistoriques de cette période. La vie d'Osman est présentée en trois époques : Osmancık, Osman Beg et Osman Gazi Han. La description de l'évolution du personnage va de pair avec le changement de la société, qui passe de l'état de tribu à celui d'État, puis de noyau d'Empire. Toute la première partie du roman décrit les conflits intérieurs d'Osman et ses questionnements sur les origines et l'avenir de son groupe. Venu en Asie Mineure avec son père Süleyman şah et ses deux frères Sunkur Tekin et Gündoğdu, Ertuğrul s'est établi à Söğüt, tandis que ses deux frères et son père ont décidé de repartir dans leur pays d'origine. Mais Süleyman se noya en traversant l'Euphrate et les frères continuèrent vers le Turkestan. C'est du moins ce que rapporte la légende et c'est cette histoire, cette mémoire que cherche à connaître l'Osmancik de Tarık Buğra pour comprendre les raisons des migrations. À travers les récits qu'il entend, il remonte peu à peu le cours du temps jusqu'aux ancêtres les plus anciens. C'est grâce à l'enseignement du şeyh Ede Bali, l'influent chef spirituel de la région, qu'Osman pourra intérioriser le sens du pouvoir pour ajuster son destin individuel au sens du destin collectif. Dans cette quête, son amour pour Malhun Hatun, la fille du şeyh, va devenir la condition indispensable, la clef de la réalisation de son destin. Le signe qu'il a atteint sa personnalité, c'est le rêve où il voit un chêne immense sortir de sa poitrine et étendre ses branches sur le monde. Le şeyh Ede Bali, qui veille à ce que la société soit correctement orientée par un dirigeant qui forme un tout avec la structure sociale, sait désormais qu'Osman est prêt pour diriger les tribus Oğuz. Par la suite, les événements sont déterminés de manière chronologique. Le passage d'un ordre tribal à un ordre étatique se concrétise par l'élargissement des lieux où vivent les 
Turkmènes et la conquête de territoires, condition pour être un État, va faire d'Osman Beg Osman Gazi Han.

La trame historique des deux romans se fonde sur des jalons attestés de l'histoire ottomane, des faits et des personnages mentionnés depuis les plus anciennes chroniques. D'après les témoignages et leurs notes de travail, on sait que Kemal Tahir et Tarık Buğra ont lu les sources turques et étrangères: des orientalistes comme H.A. Gibbons, Hammer, Paul Wittek d'une part, des représentants de la tradition historique ottomane comme Aşı Paşazâde, Ibn-i Kemal, Hoca Sadeddin, Yazıcızâde, Hayrullah Efendi d'autre part, et des historiens turcs contemporains comme Uzunçarşll, Fuad Köprülü et Halil Inalcık. Dans son ambition de concilier la mission du scientifique et de l'homme de lettres dans ses romans, Kemal Tahir surtout a effectué un impressionnant travail de recherches ${ }^{10}$.

\section{La question des sources dans Devlet Ana et Osmancık}

23 La période de la fondation se singularise par des conditions d'études particulières: alors que les sources se multiplieront pour les périodes ultérieures, l'obscurité des débuts rend difficile le travail de l'historien. Les sources de la fin du XIIIe et du début du XIV ${ }^{e}$ siècle, où l'État ottoman apparaît sur la scène de l'histoire, sont dues à des chroniqueurs byzantins (Pachymères, Nicephoras et Cantacuzène) et des géographes et voyageurs arabes (Ibn Batuta, Ibn Said, El Umarî). Mais les données relatives à l'émirat d'Osman dans ces écrits sont très peu nombreuses. Il n'y a aucune histoire turque écrite dans les premières années de l'État ottoman. Les sources ottomanes les plus récentes datent $\mathrm{du} \mathrm{XV}^{\mathrm{e}}$ siècle et si elles donnent des informations détaillées sur l'histoire ottomane des débuts, ces chroniques écrites par Neşrî, Oruç, et surtout Aşıkpaşazâde, placent l'historien contemporain face à la question de leur fiabilité et de leur interprétation.

La polémique ayant opposé Colin Imber et Halil Inalcık, dans les années 80, illustre parfaitement le sujet. Colin Imber affiche un scepticisme absolu. Selon lui, la plupart des personnages historiques mentionnés dans les chroniques ottomanes relatives à la période d'Osman Bey n'ont pas réellement existé et ne sont rien d'autre que des constructions fictives issues du folklore et de l'étymologie populaires et il affirme que "la meilleure chose que puisse faire un historien contemporain, c'est d'admettre que le début de l'Empire ottoman est un trou noir. Chaque tentative visant à combler ce vide est uniquement vouée à augmenter le nombre des récits inventés. $»^{11}$. Pour Halil Inalcik au contraire, bien que les sources traditionnelles relatives à la première période ottomane soient remplies de récits inauthentiques et rédigés à des périodes ultérieures, souvent dans le but de légitimer le pouvoir ottoman, les considérer comme de pures légendes et des mythes absolus, les rejeter en bloc à cause de ces ajouts, est une attitude qui induit en erreur. Les sources, critiquées par Imber, renferment selon Inalc1k des informations précieuses à condition de les analyser avec précaution ${ }^{12}$.

Dans Devlet Ana et Osmancık, il est intéressant d'analyser les sources que les auteurs ont privilégiées et les éléments qu'ils ont choisi de mettre en lumière ou de laisser dans l'ombre. Si les variantes que l'on peut relever entre les deux romans correspondent peut-être à un vide ou à des divergences d'informations, et à la difficulté d'établir une vérité historique indubitable, la liberté ainsi offerte au champ littéraire révèle, de façon plus essentielle, les choix et le commentaire des deux auteurs concernant une période 
qu'ils ne traitent jamais comme un décor, mais dont ils cherchent à comprendre et à exprimer les dynamiques profondes. Très conscients des questions de l'objectivité et du réalisme qu'impliquent l'approche historique, des impératifs du roman et de la responsabilité de l'intellectuel, surtout lorsqu'il s'agit de raconter une période reculée et méconnue d'une société, Kemal Tahir et Tarık Buğra revendiquent, en tant qu'artistes cette fois, la nécessité de mettre en œuvre leur propre vision du monde en soulignant que ce n'est qu'avec la force romanesque de l'écrivain que les documents gagnent du sens et qu'une pensée systématique est plus importante que le document historique, qui n'est pas en soi garant de vérité. Dans ce sens, ils présentent les erreurs historiques dont ils sont accusés comme la preuve de la cohérence de leur vision romanesque. Une cohérence qui leur donne le droit de travailler le matériau historique dans le sens requis par leur œuvre.

Le premier point de contestation dans les sources concerne la datation, toujours problématique et relevant la plupart du temps de l'hypothèse. Chacun à leur manière, les deux auteurs cherchent une voie de solution. Ainsi, la différence de quelques années dans le choix du segment historique que l'on observe entre les deux œuvres est déjà un indice de la pensée des auteurs. En prenant comme référence l'année 1290 (date admise de la succession d'Osman à Ertuğrul), Kemal Tahir se donne la liberté de concentrer en quelques mois des événements, qui selon Aşıkpaşazâde, s’échelonnent de 1283 à 1299. 1299 étant, selon certaines sources, l'année de la succession d'Osman à son père et selon d'autres, celle de l'obtention par Osman de l'indépendance, consécutive à la mort du sultan seldjoukide de Konya. Si la dimension temporelle garde une certaine élasticité dans Devlet Ana, c'est que Kemal Tahir échange le terme de " période » contre celui de « dynamique »: "Dans mon roman, je ne raconte pas telle ou telle période historique; j'essaie de montrer la dynamique d'une société qui se reflète d'une époque à une autre. [...] Le changement que j'ai opéré à l'encontre de l'histoire consiste à avoir condensé sur un an seulement une période d'une dizaine d'années. Ce n'est pas grand-chose, cela ne change rien, cela concentre simplement. En fait, ce qui est important, c'est de ne pas changer les faits et je n'ai pas été obligé de le faire ${ }^{13}$. "

Dans Osmancık aucune date n'est spécifiée. En prenant comme limite temporelle la mort d'Osman, qu'il fait coïncider avec la prise de Bursa par son fils Orhan, Tarık Buğra nous indique que le roman se passe dans le quart ou le demi-siècle précédant 1326. L'auteur suit ainsi certaines chroniques, comme celle du voyageur arabe Ibn Battuta, qui datent la mort d'Osman en 1326, alors que d'autres prétendent qu'il meurt vraisemblablement entre septembre 1323 et mars 1324. En procédant de la sorte, l'auteur exprime un thème qui lui est cher: celui de la continuité dans le temps et de l'expansion dans l'espace. La mort du premier sultan, fondateur de la dynastie des Ottomans, comme un grain tombé en terre, est ainsi le symbole de la naissance d'une nouvelle ère.

Un autre motif dominant est le thème du leader charismatique. Dans les chroniques classiques, le şeyh Ede Bali est aux côtés d'Osman, de la même manière qu'Akșemseddin sera aux côtés de Mehmet le Conquérant. Ces personnages éminents soutiennent les personnalités qui ont fondé l'État et leur donnent de la densité. Ce facteur dont Fuad Köprülü montre l'importance déterminante, Tarık Buğra le reprend pour concorder avec la tradition historique classique. Les conseils d'Ede Bali, des épisodes comme la nuit blanche passée à lire le Coran par Osman lors d'une visite au tekke, et le célèbre rêve du chêne sortant de sa poitrine et étendant ses branches sur le monde, sont autant de faits légendaires qui se retrouvent dans l'histoire de l'Islam. Cependant, comme il 
n'existe pas de réelles biographies de sultans, hormis quelques textes un peu secs à visée surtout hagiographique, de réelles biographies de sultans, l'un des attraits essentiels du roman de Tarık Buğra, c'est l'approche humaine du personnage d'Osman. Alors que dans Devlet Ana, Kemal Tahir laisse Osman plutôt à l'arrière-plan derrière une multitude de personnages incarnant les différentes forces en présence de l'époque, Tarık Buğra donne la priorité à l'analyse du monde intérieur de son héros. Il s'est penché sur la formation de la personnalité individuelle et politique d'Osman, et sur les relations réciproques entre les faits sociohistoriques de cette période et le développement de sa dimension de leader. La genèse de l'Empire se trouve donc pour Tarık Buğra dans cette convergence entre l'évolution de la sphère sociale et celle d'un individu. Une convergence qui s'effectue sous la conduite du religieux dont le rôle est de garantir et de perpétuer la légitimité sacrée du pouvoir. Ainsi, à travers les questionnements du héros en quête de lui-même, Tarık Buğra accorde une place centrale à la généalogie d'Osman alors que Kemal Tahir n'y fait allusion que de manière elliptique à travers les paroles du şeyh Ede Bali rappelant à Osman les dernières volontés de son père ${ }^{14}$. Tarık Buğra s'appuie sur la tradition officielle ottomane qui fait d'Osman le descendant de la 52e génération de Noé par un ancêtre mythique, Oğuz Khan, prédécesseur d'un non moins mythique Kayı Khan, fédérateur des tribus Oğuz. Cette descendance légendaire, enracinée dans le passé oğuz et improbable selon Paul Wittek ${ }^{15}$, est l'interprétation que privilégie Tarık Buğra, car cette descendance est montrée comme prédestinée à porter, de manière intrinsèque, les germes de l'Empire.

Dans ce sens, le mariage d'Osman avec la fille du şeyh qui, par ses conséquences politiques et son potentiel romanesque, est l'un des thèmes centraux des deux romans n'est pas traité de la même manière par les deux écrivains. Les chroniqueurs font généralement état de deux mariages d'Osman. S'ils restent plutôt confus sur les noms de ces deux épouses, la première, Mal Hatun est présentée comme la fille d'un bey turkmène, Ömer Bey, et la mère d'Orhan. La seconde épouse d'Osman est la fille du şeyh Ede Bali, Balâ Hatun ou Balâhun. Certains historiens écrivent que la fille du şeyh Ede Bali est Mal Hatun, d'autres qu'il s'agit de Balâ Hatun. Kemal Tahir conserve la version des deux mariages, en donnant le nom de Malhun Hatun à la mère d'Orhan et de Balkız à la fille du şeyh. Quant à Tarık Buğra, il choisit délibérément une version qui lui sera reprochée comme une erreur historique grossière. Il parle d'un mariage unique d'Osman avec Malhun Hatun, qui dans ce roman est à la fois la fille du şeyh Ede Bali et la mère d'Orhan. Le successeur d'Osman se retrouve ainsi placé dans une lignée qui s'ancre dans le divin.

De manière générale, Tarık Buğra opte pour des facteurs symboliquement marquants même dans sa description de la vie sociale de l'époque. Plus qu'aux conditions matérielles, il donne de l'importance à la mission des Kayı expliquée par le şeyh Ede Bali. Le destin des Kayı puis des Ottomans est de renverser les trônes et d'apporter la justice. Et en réunissant les trois principes sacrés de l'État, de la communauté et de la religion sous la bannière ottomane, Tarık Buğra rappelle d'une certaine manière que cette bannière a préparé pendant des siècles le terrain au califat. Kemal Tahir, quant à lui, parle en détail du système de l'ahilik auquel il donne le nom d'Ortak ${ }^{16}$, un système que Köprülü a décrit comme une organisation de solidarité économique et politique répandue dans tout le monde musulman. Il fait, de plus, un tableau qui mêle l'héritage seldjoukide avec la culture antique anatolienne, le chamanisme, l'alevismebektachisme et le platonisme, ce qui justifie l'étonnante apparition du poète Yunus 
Emre dans le roman. Et c'est à ce personnage représentant d'un Islam hétérodoxe que revient de raconter le rêve prémonitoire du şeyh Ede Bali.

Devlet Ana et Osmancık appartiennent sans conteste à la catégorie du roman historique. En s'appuyant sur les données historiques relatives au contexte de l'Anatolie à l'aube du XIV ${ }^{e}$ siècle, Kemal Tahir et Tarık Buğra, par des voies différentes, mettent en évidence que l'effondrement politique, économique et moral des deux géants seldjoukide et byzantin en Anatolie est un facteur qui a contribué à l'émergence des Kayı. Kemal Tahir, avec une méthode qui concorde avec le déterminisme de Köprülü, analyse tous les éléments politiques, économiques et sociaux qui ont contribué à l'émergence de la tribu des Kayı et à la fondation d'un État qu'il analyse comme le résultat d'une obligation politique. Tarık Buğra, qui a tendance à conserver les événements tels qu'ils sont décrits par les chroniqueurs, a essayé de comprendre les symboles et les éléments mythiques de cette histoire qui semble aller dans le sens de sa propre logique et s'inscrit dans le fil de la tradition historique officielle ottomane. En insistant sur l'évolution psychologique et spirituelle d'Osman sous la direction du şeyh Ede Bali, il montre que les Kayı sont poussés par un destin inéluctable qui s'enracine dans un dessein divin. Cependant, en recourant à des éléments formels relevant de l'épopée et du mythe, ces deux œuvres ne semblent pas satisfaire pour la critique, les critères du roman historique réaliste.

\section{Des modèles formels relevant du mythe}

La vision historique et les conceptions romanesques des deux auteurs déterminent bien entendu l'angle de leur œuvre: opposition Orient Occident dans Devlet Ana et leadership lié au thème de la prédestination dans Osmancık. Cette approche porterait à penser que Tarık Buğra, par l'importance qu'il accorde au contenu symbolique et sacré, est le plus prédisposé à recourir au mythe. La place du destin et du temps, l'emploi de motifs de conte comme le Phoenix ou le Mont Kaf en référence au récit mystique de "Attar ", Le langage des Oiseaux, ou encore la citation de versets du Coran, installent immédiatement une atmosphère poétique et de légende. Mais à la lumière des analyses de Berna Moran, qui qualifie Devlet Ana de «romance» dans le sens de la littérature chevaleresque du Moyen Âge ${ }^{17}$, il apparaît que dans Devlet Ana également, l'aspect épique est dominant. En mêlant les thèmes de l'héroïsme et de l'amour, Kemal Tahir fait référence aux récits de Dede Korkut, aux contes populaires des aşık (bardes populaires itinérants) et à Evliya Çelebi, de manière évidente dans les noms donnés au couple Kerim et Aslı (en référence à la légende de Kerem et Asli) et en filigrane dans le style même du texte: «Lorsque je parlerai des groupes de guerriers ottomans, j'utiliserai le style de Dede Korkut. Lorsque je ferai parler le groupe des dirigeants, je crois qu'Evliya Çelebi sera le bienvenu $»^{18}$.

Manifestes dans les références littéraires employées par les deux auteurs, les aspects du mythe sous-tendent la structure même des œuvres. Au niveau de la composition, on retrouve dans les deux romans des modèles formels typiques de l'épopée et du conte, dont les racines relèvent du mythe. Le schéma de ce type de récit, construit selon les trois étapes de la séparation et/ou de la perte, des épreuves, et de la renaissance, se retrouve à plusieurs niveaux dans Devlet Ana et Osmancik et le parcours de certains personnages présente tous les aspects symboliques des rites de passage propres au 
chemin initiatique. Dans Osmancık, la quête revient au personnage d'Osman et à Kerim dans Devlet Ana.

\section{Il était une fois un royaume} des facteurs externes et internes ayant une répercussion sur le sort des Kayl. Mais une différence frappante ressort du tableau que brossent les deux auteurs. La situation initiale décrite dans Devlet Ana donne l'image d'un royaume où s'abattent les fléaux et Ertuğrul, âgé et malade, est à l'image du pays. En fait, dans le roman, la vieillesse et la maladie d'Ertuğrul Bey ne sont pas ouvertement montrées comme les raisons de l'état de famine et de pauvreté dans lequel est tombé Söğüt mais il est signifié que la maladie de ce dernier a commencé en même temps que la pauvreté de Söğüt, et de nombreuses pages du roman ont trait au manque et à la nostalgie de l'abondance passée. Ainsi, dans un ordre où le pouvoir est très personnalisé, la tribu est menacée de chaos, comme un édifice ayant perdu sa clef de voûte. Seule l'investiture de son successeur peut redonner vie à ce corps en voie de dépérissement. Et c'est seulement après le choix d'Osman à la succession, que Söğüt retrouvera la prospérité.

Chez Tarık Buğra au contraire, Ertuğrul incarne totalement le pouvoir et son sabre, symbole de force placée sous la conduite de la sagesse, est une image récurrente qui aiguise les questionnements de son fils Osman. La situation initiale de manque provient de l'attitude d'Osman, qui refuse et fuit la responsabilité de ce pouvoir. Même si la situation économique du beylik est fragile, ce sont des images d'abondance qui prédominent. Par l'intermédiaire du personnage d'Osman, qui contemple les migrations saisonnières, l'auteur établit une similitude entre le flux du temps et le flux des populations qui s'écoulent comme un fleuve intarissable : «Des dizaines de milliers de moutons, des milliers de boufs, de vaches, de chevaux et des dizaines de milliers de personnes! [...] On passait par des fleuves aussi grands que des océans, des cimes où jamais la neige ne manquait, des lacs qui ne voyaient jamais l'ombre, des plaines sans fin. Tant de filles, tant de fils disséminés le long de ces migrations avaient grandi, s'étaient mariés, et étaient devenus des mères et des pères ${ }^{19}$. » Alors que l'affluence de populations dans Devlet Ana, est décrite au départ comme un facteur de danger et d'accroissement de la pauvreté, ce même élément est très vite montré comme porteur d'un accroissement de force poussant à l'expansion et le signe d'une bénédiction dans Osmanckk. Et loin des sombres marécages qui sont un lieu fondamental de l'action et fortement symbolique dans Devlet Ana, Tarık Buğra décrit "les symphonies de couleurs paradisiaques des rêves" ${ }^{20} \mathrm{du}$ flot humain s'avançant sous les yeux d'Osman. Le danger pour les tribus provient des luttes intérieures d'Osman qui les placent en position d'attente et pourraient les priver de leur destin.

\section{Les personnages de la quête}

Dans l'œuvre de Tarık Buğra, le parcours d'Osman, une longue quête de sa personnalité et d'une destinée qu'il doit ajuster à celle de la société est construit en trois étapes : Osmancık, Osman Beg et Osman Gazi Han. Ce schéma ternaire marque trois grands niveaux de conscience de sa vie, ainsi que trois stades essentiels de l'évolution de la société. La période Osmancık, c'est la jeunesse d'Osman, caractérisée par le fait qu'il n'est pas maitre de sa force, mais lui est soumis. Il est décrit comme ne vivant que par

Cahiers balkaniques, 36-37| 2008 
et pour son orgueil. Ainsi, toute sa force est tournée vers l'extérieur. En réagissant immédiatement par la colère, comme dans l'épisode de son conflit avec Kalanoz, Osman représente ainsi le type même du gazi, comptant sur sa force physique, réagissant de manière instinctive sans pensée politique à long terme. Cette violence intérieure le met en rupture avec sa société. L'individu tout comme le groupe souffrent de ce déséquilibre qui demande à être rétabli. Sous la conduite d'un guide, le şeyh Ede Bali et ses auxiliaires, cette première phase d'apprentissage passe par la mort symbolique d'Osmancık, qui à travers l'épreuve de la conquête amoureuse, parvient à se transformer pour devenir Osman Beg. Cette deuxième étape est celle de la maturité individuelle et politique, capable d'entraîner le groupe qui évolue avec son dirigeant et cela s'exprime par les conquêtes. Enfin, la mort d'Osman Gazi Han, sur laquelle s'ouvre et se referme le roman, n'est pas une fin, mais un couronnement. L'achèvement d'une vie qui se poursuit à travers sa descendance.

Dans Devlet Ana, le même schéma se retrouve dans le parcours du personnage de Kerim. Tandis que Tarık Buğra, à travers le personnage d'Osman, opère une synthèse entre drame social et drame individuel, Kemal Tahir organise son roman selon deux axes : l'histoire de Kerim en quête des meurtriers de son frère, et celle de l'élargissement des territoires et de la fondation du noyau de l'État ottoman par les Turkmènes du village de Söğüt. L'une sociale, l'autre individuelle, ces deux histoires sont imbriquées, car les ennemis de Söğüt et de Kerim sont les mêmes. L'élément narratif concernant les Turkmènes, c'est l'histoire, sous la direction d'Osman, du passage du manque à l'abondance et avec l'élargissement de leur territoire, du passage de la condition de tribu à celle d'État. C'est l'histoire de la réussite au niveau collectif de ce qui est raconté au niveau individuel dans l'histoire de Kerim.

L'histoire de cet adolescent, qui entre en scène par le biais de l'imitation d'un rite d'initiation dans l'organisation des Ahi, est le récit de son initiation selon les trois étapes traditionnelles, séparation, épreuves, retour. Malgré l'opposition de sa mère, l'intraitable Bacı Bey, dont le surnom Devlet Ana (Mère Patrie) donne son titre à l'œuvre, Kerim se destine à être mollah. Mais le meurtre de son frère par les ennemis de Söğüt vient réduire ses projets à néant. Bacı Bey brûle les livres et le saz de Kerim et l'oblige à coups de fouet à endosser le rôle de guerrier pour venger la mort de son frère. Kerim Çelebi devient Kerim Can et commence la deuxième phase de son initiation. Il s'éprouve dans l'art de la guerre, retrouve les meurtriers de son frère, et doit s'affronter à des ennemis, qui par leur noirceur d'âme, ont toutes les caractéristiques du monstre souterrain. Il sort vainqueur de l'épreuve en emportant sa part de trésor (une dizaine de manuscrits de grande valeur), gagne l'amour de la fille qu'il aime et est acclamé pour avoir montré sa bravoure. Cette seconde étape s'achève dans les toutes dernières pages du roman où convergent l'histoire individuelle et l'histoire collective. Les Turcs ont vaincu leurs ennemis et Kerim a achevé sa mission en vengeant la mort de son frère. Il obtient l'autorisation d'Osman de partir étudier à Bilecik dans la medresse du şeyh Ede Bali et sa mère n'est plus en mesure de s'opposer à lui. L'enfant Kerim est mort et le fouet qui symbolise l'autorité de son père est entre ses mains. À travers les épreuves il a trouvé sa place dans la société des hommes et Kerim Can peut reprendre son nom. La dernière page de Devlet Ana se referme sur Kerim Çelebi plongé dans la lecture du Siyasetnâme.

On retrouve dans ce roman une atmosphère de roman d'aventures et le comique n'est pas absent, surtout dans le trait grotesque dont use Kemal Tahir dans sa description de 
l'Occident, notamment à travers Notüs Gladyüs et le moine Benito, un duo qui semble rassembler toutes les calamités de la nature humaine. Avec cette approche caricaturale, on est bien loin de ce que l'on peut attendre du réalisme littéraire, et surtout de la dimension psychologique sensible de Tarık Buğra. Mais le thème chevaleresque de ces romans semble pousser les auteurs vers d'autres formes que celles du roman réaliste. Par l'exemplarité des héros, les vertus qui font la tradition et la manière de les mettre en pratique, Devlet Ana et Osmancık renouent plutôt avec une dimension pédagogique présente dans l'épopée. En faisant entrer dans les structures narratives des traits qui relèvent $d u$ mythe et sont composés des thèmes de l'amour et de l'aventure, en $\mathrm{y}$ ajoutant leurs conceptions relatives au peuple turc, à son univers, à sa vision des choses, à ses aptitudes et ses vertus, les deux auteurs réactualisent en somme des modèles formels qui ont été expérimentés depuis des siècles.

\section{Une allégorie national(ist)e}

\section{Supériorité d'un ordre reposant sur la justice et la tolérance}

En analysant les succès politiques des Turcs, les deux auteurs ne sont pas loin de l'enthousiasme de Namık Kemal qui qualifiait la naissance de l'Empire ottoman de miracle.

41 Tarık Buğra déclare que le secret de la réussite des Turcs réside, non dans la force, mais dans la tolérance et leur attachement au droit et à la justice. Pour Kemal Tahir, le facteur principal de ce succès c'est le don et le génie des Turcs à fonder et faire perdurer des États. De plus, sa vision historique, même si elle se fonde sur le principe marxiste de classes va totalement dans le sens de Tarık Buğra: «Comme il n'y a pas de classes dans les sociétés orientales, dit-il, l'État repose seulement sur la justice ${ }^{21}$.» En décrivant le mode de vie de la société à cette époque, à l'intérieur de la tribu et dans ses relations avec son environnement, les deux romans s'accordent pour faire ressortir les vertus de la justice et de la tolérance propres aux Kayl, des vertus qui s'exercent aussi bien dans le domaine économique, religieux, politique que dans le domaine de l'amour et de la famille. À l'encontre des théories historiques décrivant les tribus à l'origine des Ottomans comme un ordre économique fondé sur la razzia, Kemal Tahir et Tarık Buğra montrent qu'il s'agit d'un ordre certes guerrier, mais pacifique par essence, et que ce soit dans l'acception de l'Islam ou du socialisme, ils font des premiers Ottomans un parfait modèle communautaire.

Du point de vue économique, les Kayı veillent au partage des biens entre le peuple et les guerriers et les beys turkmènes sont décrits comme ne possédant rien personnellement à leur mort. Le système de l'ahilik empêche l'accumulation individuelle des richesses et la formation d'une classe possédante. C'est la raison pour laquelle, selon Kemal Tahir, la société ottomane est une société sans classes, radicalement différente de la société occidentale, issue du féodalisme fondé sur l'esclavage. Une thèse qu'il incarne dans le personnage du prisonnier de guerre Kurt Ali, dont la situation symbolise toute la dégradation et l'inhumanité d'un ordre occidental barbare. Fait prisonnier lors d'une bataille navale, cet ancien lieutenant de vaisseau se déplace dans les confins avec des chaînes pour mendier le prix de sa libération et trouve auprès des Turcs la générosité qui fait défaut aux Occidentaux, montrés comme avides et brutaux. Le rapport de maitre à esclave apparaît encore dans la relation entre Mavro et le gouverneur chrétien 
Filatyos, qui le fait battre pour lui faire avouer le meurtre de Demircan. Filatyos est arrêté par le sabre de Bacı Bey qui prend Mavro sous sa protection. Une fois réfugié chez les Turkmènes, Mavro qui devient un vrai guerrier, mesure à quel point sa société s'est dégradée et menacé d'être fait prisonnier par son ancien maittre, il choisit de se convertir à l'Islam, au sens de se faire Turc.

Avec la conquête de nouveaux territoires, la domination des Turcs est facilement acceptée par les populations chrétiennes qui les accueillent même parfois en libérateurs contre le pouvoir arbitraire des chrétiens. Et l'Islam qui se propage n'est pas perçu comme une religion ennemie, mais comme la garantie d'une justice qui n'existe pas dans le monde chrétien. Dans Osmancık également, un personnage symbolise la prise de conscience de la contradiction qui existe entre les deux mondes. En se convertissant de son plein gré à l'Islam, Köse Mihail (Michel le Glabre) fait le choix d'une communauté où règnent la solidarité et la défense des valeurs humaines, sans discrimination de religion ou d'ethnie.

Concernant la religion, Kemal Tahir gomme au maximum le facteur religieux comme motivation des conquêtes, au point de faire d'Osman un fervent défenseur de la laïcité. La vision plus spirituelle de Tarık Buğra accorde aux dirigeants spirituels un rôle fondamental dans l'orientation du gouvernement. Représentants d'un Islam purement orthodoxe, ce sont eux qui veillent à l'essence sacrée du pouvoir et ils sont tous irréprochables. Chez Kemal Tahir au contraire, qui s'attache à montrer la diversité des moines, derviches et abdas sillonnant l'Anatolie, le tableau est plus vaste, mais le scepticisme mordant. Les religieux sont le plus souvent décrits comme profitant de leur statut pour réaliser des profits crapuleux. La loi canonique par exemple ne peut être à elle seule une garante absolue de la justice, puisque Dündar Alp, le frère d'Ertuğrul veut l'utiliser à son profit. La corruption est dans l'humain et seul le combat moral peut en éradiquer les germes. Pour Kemal Tahir, justice et tolérance sont des valeurs en soi, et ces valeurs prennent leur source dans la nature même des Turcs par opposition à la sauvagerie des Occidentaux ou d'autres ordres sociaux.

En revanche, comme chez Tarık Buğra, le şeyh Ede Bali dans Devlet Ana est un représentant du monde de la connaissance. Premier aux côtés d'Osman dans la direction de l'État, il s'installera à Bilecik après la prise de la ville, Bilecik devenue importante du point de vue géopolitique maintenant que les Turcs ont décidé de s'orienter vers l'Ouest. Avec l'organisation de l'État, il est nécessaire d'éduquer le pays pour lui donner les cadres religieux et juridiques nécessaires à sa direction. Le personnage de Kerim est intéressant de ce point de vue. Confronté aux quolibets à cause de sa préférence de la voie du mollah à celle du guerrier, (pour sa mère et sa petite amie, ce sont les lâches, les infirmes, ceux qui ne servent à rien qui choisissent d'être hodja), Kerim a pu faire des études grâce à la tolérance et à la vision éclairée d'Ertuğrul. Le pays est en effet voué à entrer dans sa dimension sédentaire et étatique, et il aura besoin de gens instruits et compétents dans divers domaines. Après la prise de plusieurs forteresses et l'orientation des Ottomans vers Iznik et Bursa, la possibilité pour Kerim d'être mollah apparaît de nouveau. La conception de l'héroïsme et du mérite ne repose plus seulement sur l'idéal du guerrier, et Kemal Tahir opère à travers ce personnage une synthèse entre l'épée et la plume. Le parcours de Kerim est aussi annonciateur du nouvel ordre de l'État ottoman où l'organisation de la justice vient au même rang que celle de la guerre. La même conscience et l'orientation vers un ordre sédentaire sont également décrites dans Osmancık. 

démocratiques des Kayı, notamment lors du choix d'Osman à la succession. Un choix fondé sur la personnalité d'Osman et non sur des principes de dynastie, de rang ou de richesse. Les conflits pour le pouvoir surgissent entre Osman et son oncle Dündar Alp, à peu près l'unique élément négatif de la tribu. Kemal Tahir en fait un personnage célèbre pour sa cupidité et sa vénalité, un opportuniste qui trouve ses alliés parmi les derviches et dans ses relations commerciales avec les Byzantins. Osman en revanche veut défendre et répandre l'ordre de justice instauré par son père. Dans Devlet Ana, tous les problèmes intérieurs et extérieurs de l'émirat sont discutés sur la place du village où se réunissent le peuple et tous les responsables de la société et du gouvernement, donnant ainsi l'image d'une vraie démocratie. Chez Tarık Buğra, le şeyh Ede Bali est au cœur de la décision, mais en tant que guide seulement. À la différence du roman de Kemal Tahir, les décisions sont prises à huis clos, mais cette modalité ne fait que souligner un peu plus le lien de confiance entre peuple et dirigeants. De nombreuses pages d'Osmancik sont consacrées aux discussions du conseil où Osman utilise systématiquement le principe de consultation. Dans les deux romans d'autre part, le choix de porter les conquêtes vers l'Ouest est constamment souligné comme le signe de l'intelligence politique d'Osman : « Je vais m'éloigner de l'Anatolie pour l'instant. Les Mongols sont sur le point d'arriver. Notre terre d'Anatolie ne peut pas s'entendre avec l'ordre mongol. Parce qu'elle ne s'est pas entendue avec l'ancien ordre grec ni l'ordre romain. Nos beylik gazi n'ont qu'à se battre pour mettre la main sur Konya. Qu'ils s'entretuent les uns les autres, ils me faciliteront le travail. Les possesseurs des terres fertiles, voilà ce qui est utile à l'Anatolie. C'est cela la source d'un peuple qui ne s'éteint pas, et l'aptitude du peuple ce n'est pas d'être paysan, c'est de fonder un État ${ }^{22}$. " Cet élément a une grande importance, car il s'oppose aux clichés tendant à montrer les guerriers turcs comme des barbares n'ayant aucune intelligence politique à long terme. Finalement, les deux auteurs s'appliquent à démontrer que le pouvoir ottoman n'était pas despotique et que tous les principes de la démocratie existaient depuis les origines dans le monde ottoman, avant même qu'ils ne soient découverts par l'Occident. fois montrés supérieurs aux Occidentaux. L'amour est défendu en tant que valeur fondatrice de l'individu et de la société, et plusieurs combats sont d'ailleurs liés à l'amour d'une femme. L'aspect moral est très appuyé chez Tarık Buğra qui fait de la conquête de Malhun Hatun par Osman, l'équivalent d'une quête mystique et enracine sa descendance dans les liens sacrés du mariage, nous l'avons vu. Si l'amour est aussi une valeur défendue dans Devlet Ana, Kemal Tahir est beaucoup plus réaliste sur la question et la sexualité occupe une place importante. Il est fait mention de l'homosexualité, de la pédophilie tolérée par d'autres groupes (et pratiquée par Dündar) et de la polygamie, dont on connaît la place dans le développement de l'Empire. Osman a deux épouses et à la fin du roman apparaît une concubine. Mais l'auteur montre que la femme est respectée, en contraste avec les appétits bestiaux et la pensée arriérée que prête Kemal Tahir aux Occidentaux, notamment à travers le personnage du chevalier Notüs Gladyüs et la critique du système de la dot. Le rôle accordé aux femmes par Kemal Tahir, avec les guerrières turkmènes de Bacı Bey, capables de mettre en déroute les plans ennemis et de prendre une forteresse, rappelle le statut particulier des femmes dans l'ensemble turco-mongol, en marge du contexte musulman et méditerranéen, tel qu'il apparaît dans Dede Korkut. Le surnom de Bacı Bey, 
Devlet Ana, semble incarner le thème de la nation soldat qui était apparu chez Namık Kemal et sera par la suite l'un des thèmes majeurs du nationalisme.

Enfin, les deux auteurs montrent que la bravoure des Turcs leur attire constamment les faveurs des femmes chrétiennes, un facteur dont la dimension politique est considérable. Dans Osmancık, l'amour d'une Grecque pour un guerrier d'Osman ouvre aux Turcs les portes d'une forteresse. Le thème de l'exogamie illustre aussi la tolérance des Turcs, ouverts à l'intégration des populations hétérogènes. La description de cette dynamique d'absorption culturelle, toujours librement choisie par les nouveaux arrivants, semble vouloir faire pendant aux mouvements nationalistes séparatistes des $\mathrm{XIX}^{\mathrm{e}}$ et $\mathrm{XX}^{\mathrm{e}}$ siècles, où les non Turcs voulaient se libérer du joug ottoman. Ainsi pour Kemal Tahir comme pour Tarık Buğra, l'expansion turque est aussi bien politique, militaire que culturelle, mais surtout, est suggérée l'idée que les Turcs étaient porteurs d'une mission civilisatrice. Dans cette démarche, on retrouve les préceptes de l'ottomanisme qui s'est employé à intégrer les diverses communautés ethniques et religieuses, mais une idéologie qui a finalement jeté les bases du nationalisme.

Malgré les différences concernant l'approche de la religion, les deux auteurs montrent clairement que l'ordre musulman ou turc s'oppose pied à pied aux autres ordres sociaux (byzantin, seldjoukide, mongol) et surtout au monde occidental qui cherche à le détruire. Cet ordre musulman ou turc est supérieur en tout au monde chrétien occidental. Supériorité dans le domaine militaire, moral, social, culturel, technique et politique. Au total, les deux romans prennent systématiquement le contre-pied de tous les points montrés comme les éléments ayant contribué au retard et à la chute de l'Empire ottoman. Ainsi, les premiers temps de l'Empire ottoman apparaissent comme l'idylle sociale du Moyen Âge, un âge d'or emporté par les courants de l'occidentalisation.

\section{Défense d'une identité turque et d'un art national}

50 À travers ces deux romans se révèle une problématique commune : celle de répondre au besoin de retrouver les racines d'une histoire nationale qui soit valorisante et en plongeant dans une recherche historique sur son passé, Kemal Tahir et Tarık Buğra ont cherché à offrir à la Turquie une identité originelle qu'ils jugeaient sinon perdue, du moins fourvoyée dans les chemins de l'histoire et dans "l'impasse de l'Occident». Mettre en lumière les aspects positifs qui ont présidé à la fondation de l'Empire ottoman leur apparait comme une démarche essentielle pour permettre au pays de retrouver sa réelle identité. Et ce qui relève plutôt du mythe que de l'histoire dans ces romans, c'est peut-être la quête, avec les origines de l'État, de valeurs originelles d'avant la dégénérescence. Avec Osmancık, Tarık Buğra présente un type d'explication adopté depuis le $\mathrm{XV}^{\mathrm{e}}$ siècle par l'État pour affirmer la domination ottomane, et pour Murat Belge, le fait que l'auteur parle de «secret des Ottomans » après le travail d'un historien comme Fuad Köprülü, c'est le choix d'aborder le sujet comme un «miracle métaphysique » qui n'a plus rien à voir avec l'histoire ${ }^{23}$.

51 Kemal Tahir dans sa démarche n'échange pas totalement le marxisme contre l'ottomanisme, il essaie plutôt d'opérer une synthèse entre les deux. Et comme l'analyse Taner Timur, c'est justement dans son approche marxiste et sa conception du système de production de type asiatique (ATÜT), que résident les contradictions de Devlet Ana. L'ATÜT qui est un concept qui n'a pas atteint toute sa limpidité chez Marx est en fait le 
symbole d'une structure sociale primitive n'ayant pas encore atteint le développement d'une société de classes. Ce terme véhicule en fait un sens péjoratif allant de pair avec le despotisme oriental. Malgré cela, Kemal Tahir s'est chargé de la défense des sociétés orientales en essayant de les présenter comme un ordre idéal de justice. Un ordre que Marx qualifiait de despotisme devient chez Kemal Tahir la Mère Patrie et, partant du principe que l'État ottoman était le garant de la sécurité et de la liberté des personnes, il défend et loue l'ordre ottoman traditionnel comme une valeur absolue ${ }^{24}$. Les vues de Kemal Tahir lui attirèrent les mêmes accusations que celles habituellement destinées aux gens de droite, notamment celle de plaire aux fascistes et de frôler parfois le racisme.

Le but principal de Kemal Tahir lorsqu'il écrit Devlet Ana, plus encore que de faire de ce roman un nouveau champ d'application de ses idées marxistes, c'est de vaincre le complexe d'infériorité des Turcs venant de leur histoire et leur faire acquérir une confiance et une fierté nationale : "Il y a un manque de confiance, une angoisse chez les gens, on se débat dans le désespoir. Tout cela à mes yeux est un chaos d'avant la naissance. Avec mon nouveau roman, je vais essayer d'effacer ce manque d'espoir, de donner confiance à la société et de lui apporter un nouveau souffle. C'est la raison pour laquelle j'ai choisi les premières années de la fondation de l'Empire ottoman comme sujet ${ }^{25}$. » Tarık Buğra, commentant sa persévérance à écrire constamment des chroniques sur le même thème avant d'en faire un roman, exprimera son but dans les mêmes termes: "Ces écrits qui paraissent dans plusieurs revues et journaux pendant plusieurs années montrent à quel point un auteur peut être condamné à un sujet. Cette condamnation provient de l'ingratitude historique que nous connaissons tous [...], elle est née de tous les slogans qui veulent que la nation se désintègre dans un complexe d'infériorité2 ${ }^{26}$.»

C'est donc sur le terrain de la défense d'une identité nationale et par là, d'un art national, que ces deux auteurs a priori très différents dans leurs conceptions se rejoignent dans les mêmes termes. Ils pensent que c'est surtout avec les mouvements d'occidentalisation que l'ordre ottoman s'est complètement effondré et ils s'interrogent sur l'impasse culturelle dans laquelle est tombée la Turquie. Au lieu de faire évoluer la structure sociale ottomane à partir de ses propres fondements pour trouver des solutions aux problèmes de l'Empire, les classes dirigeantes ont opté pour la conduite d'une politique d'occidentalisation, qui est selon eux une politique erronée contraignant la société turque à endosser des réalités politiques et culturelles qui ne sont pas les siennes.

Dans leurs déclarations, l'un et l'autre sont extrêmement virulents sur la question de l'imitation et la critique de l'Occident. Pour Kemal Tahir le concept d'humanisme et la raison que l'Occident a brandie comme un drapeau "sont un rideau de fumée pour dissimuler l'exploitation bourgeoise qui est l'exploitation la plus éhontée du monde. Quant à l'Occidental, c'est un cannibale en cravate qui, une fois qu'il a décidé de dévorer de la chair, ressemble, pour ceux qui regardent de l'extérieur, à l'Église chrétienne qui embrasse les gens en leur faisant lever la tête, mais pour ceux qui voient l'affaire de l'intérieur, il est clair que c'est à coups de pied qu'on leur fait redresser la tête $»^{27}$. Kemal Tahir considérant comme synonymes occidentalisation et colonisation, avance que l'idéal de parvenir à la civilisation occidentale, surtout de passer à la civilisation occidentale, est encore plus destructeur que le fait même d'être occidental. Une conception qui a pu faire soupçonner Kemal Tahir d'antikémalisme et à laquelle fait écho ce texte de Tarık Buğra : «La stratégie du dehors, ses buts sont évidents : anéantir l'identité et ainsi éradiquer ce 
qui résiste. Et l'intérieur alors? C'est-à-dire la fraction coupable, orientée, qu'elle le veuille ou non, vers le même résultat que l'extérieur? Dans notre histoire artistique, littéraire, intellectuelle et politique, la fraction coupable est représentée par les intellectuels, les progressistes, les bien-pensants, les occidentalistes et louée comme telle. [...] Au sujet de la culture, ils ont considéré comme égales l'erreur et la trahison. Ils ont recherché l'issue dans l'imitation. Ils sont tombés dans le piège de considérer notre culture comme l'obstacle au progrès et à la civilisation [...] au lieu de voir à quel point ils étaient liés aux cultures naturelles des nations les plus évoluées, les plus cultivées, les plus riches et de rechercher comment les utiliser. Ces créatures qui poussent comme des champignons, ces importateurs de la culture occidentale, ces commissionnaires se sont vraiment conduits comme des barbares. Ces commissionnaires, ces réactionnaires, ces conservateurs, sectaires, ennemis de la révolution, voire fascistes, voire même racistes, sans avoir trouvé d'étalon scientifique et objectif ont agressé les travaux en art, en littérature et les visions du monde qui défendaient l'identité de la culture turque dans son approche de l'humanité orientée vers le développement ${ }^{28}$."

Le débat sur la défense d'une identité et d'un art turcs s'est manifesté dans tous les domaines de la vie politique et culturelle, notamment dans le domaine cinématographique où les thèses de Kemal Tahir ont été adoptées comme une philosophie dans les années 70 . Les défenseurs d'un cinéma nationaliste et ceux d'un cinéma national se trouvèrent des points communs sur les grandes lignes. Les deux défendaient l'existence du cinéma turc contre le cinéma étranger, l'un plus spiritualiste, l'autre plus matérialiste. La frontière entre national et nationaliste n'est donc pas toujours facile à établir. Cela dépend en fait de qui, de quel camp vient la critique. Et la critique est le plus souvent idéologique, malgré les meilleures intentions du monde comme le déplore Tarık Buğra : "j'ai essayé d'être un écrivain des droits et de la liberté pour les gens, et de l'indépendance pour les pays [...]. Osmanc1k, je l'ai voulu comme le roman de la tolérance, que je considère comme une vertu capitale... Comme je n'ai jamais suivi aucune clique, aucun parti, aucun courant, aucun slogan, je ne me suis pas seulement retrouvé seul, j'ai été traité de fasciste, de réactionnaire, de conservateur ${ }^{29}$. "

Dans une atmosphère de pensée partisane, Kemal Tahir et Tarık Buğra, se retrouvent dans une même violence de propos quand il s'agit de défendre leur position. Face aux critiques portant sur ses conceptions historiques, donc politiques, Kemal Tahir, dont l'écriture et la pensée théorique sont indissolublement liées, s'est toujours défendu en insistant sur le fait qu'il était avant tout un romancier. Quant à Tarık Buğra, en plaçant la littérature au-dessus de tout, il n'aura de cesse de pourfendre les critiques fondant leurs commentaires sur des critères idéologiques. Attaqués sur leur idéologie, les deux auteurs retournent le compliment à ceux qui les critiquent... et on ne sort pas du cercle vicieux de la polémique. L'idéologue, c'est toujours l'autre finalement. Dans un article intitulé "La culture politique ", Murat Belge s'attache à montrer que cette constante violence verbale, quand elle n'est pas physique, est précisément un héritage des Ottomans. L'ordre idéal de cet État étant la séparation du dirigeant et des dirigés, les Ottomans n'ont pas laissé d'espace à la vie politique publique. Cette conception du gouvernement et de l'État, qui s'est perpétuée dans la période républicaine, a empêché le développement d'une culture ouverte à la critique et au débat. Le face à face est forcément une guerre qui doit se terminer par l'anéantissement de l'adversaire. Murat Belge analyse ce dogmatisme de la pensée comme étant lié à la notion du sacré qui fonde la naissance de l'État ottoman. Avec des nuances, certes, les auteurs de Devlet Ana et d'Osmancık ne disent pas autre chose. Cette dimension du sacré est passée dans toutes les idéologies qui visaient précisément à sauver l'État et tous ces courants 
(ottomanisme, panislamisme, panturquisme, kémalisme) ont développé une rhétorique et une conception fondamentalistes qui ont contaminé avec le temps les autres idéologies qui commençaient à se développer en Turquie. Le socialisme et l'islamisme par exemple. Si les formes ont changé, les habitudes mentales sont restées. Et c'est bien de l'hégémonie de la vision historique à but politique que proviennent l'obsession idéologique et le fait que le libre débat commence à peine dans sa véritable dimension démocratique en Turquie.

\section{Conclusion}

Avec Devlet Ana et Osmancık, nous avons vu que l'histoire, le plus souvent évaluée au regard de sa conformité aux conceptions politiques, pose l'épineuse question de la vérité historique et de sa représentation littéraire. La différence d'approche de Kemal Tahir et de Tarık Buğra s'exprime fortement au niveau de la mise en œuvre artistique. Par son insistance à transmettre des informations historiques pour atteindre une valeur qu'il considère comme encore plus importante que l'art, Kemal Tahir a tendance à délaisser quelque peu la réalité humaine de l'individu. Surtout, son enthousiasme à rendre compte de ses découvertes historiques semble prendre le pas aussi bien sur l'aspect artistique que dialectique. En effet, il semble qu'au fil de ses recherches historiques, sa vision ne concorde plus totalement avec certains aspects du marxisme tels qu'ils s'expriment ailleurs de façon plus catégorique et dans ses derniers romans, Devlet Ana notamment, Kemal Tahir semble très éloigné de ses premières idées. Les contradictions de Kemal Tahir entre ses propos théoriques et leur mise en œuvre, les contradictions dans ses déclarations elles-mêmes sont nombreuses et c'est peut-être l'aspect le plus intéressant de cet auteur perpétuellement en quête d'un système théorique qui constitue à la fois une dynamique de pensée et un cadre jamais satisfaisant. Si Tarık Buğra aborde également les aspects politiques, historiques et sociaux dans ses œuvres, ce qui reste au premier plan "plus que les événements historiques, ce sont les gens au cœur de ces événements ${ }^{30}$. L'essentiel pour lui, c'est de pouvoir saisir les aspects immuables et universels de l'humain et ce qui le distingue d'autres écrivains turcs, c'est la place qu'il accorde à des éléments tels que le destin, le temps, les luttes intérieures et la minutie de son style. Pour Tarık Buğra, « réfléchir à la littérature et s'intéresser au sort de la Turquie, cela revient au même ${ }^{31}$. La différence d'approche et de style entre les deux auteurs a pu faire dire que de Kemal Tahir il restait les idées, et de Tarık Buğra, les œuvres ${ }^{32}$.

De l'histoire comme modèle hégémonique de pensée à l'histoire mise en doute. Cela pourrait résumer l'évolution de l'approche historique de la littérature ces dernières années. C'est seulement depuis les années 80 , et de manière plus évidente encore après 1995, que l'on assiste à un retour de l'histoire ottomane. Avec des auteurs comme Orhan Pamuk, Gürsel Korat, Ihasan Oktay Anar, Nedim Gürsel ou Ahmet Altan, les romans ayant trait à des sujets historiques de la période ottomane et seldjoukide se sont répandus de manière évidente sur le marché. Est-ce l'éloignement dans le temps qui rend le sujet plus historique, donc plus apte à être appréhendé que le présent ? Si l'histoire peut constituer une échappatoire d'un présent face auquel on manque de distance, le choix d'une période historique est souvent déterminé par sa capacité à renvoyer en écho une image du présent. La mise en perspective du temps à travers le jeu des relations entre personnages et narrateur est d'ailleurs l'un des traits 
caractéristiques des romans de la dernière génération. Dans la mise en doute de l'histoire propre à cette génération, on peut voir un sursaut de la littérature cherchant à reprendre ses droits sur l'histoire. Orhan Pamuk va jusqu'à dire qu'écrire l'Histoire, c'est tout simplement raconter des histoires.

Cette foi en la littérature ne peut cependant satisfaire l'historien. Et la période de la fondation de l'Empire ottoman, aujourd'hui, plus qu'hier, alimente de nombreux travaux. Malgré des chroniques très postérieures aux faits, de rares témoignages sujets à caution et une trame événementielle problématique sur bien des points, certaines dates et certains faits, par recoupement des sources, peuvent être solidement établis, notamment grâce à la numismatique, à l'épigraphie, à des registres de recensement de la fin du $\mathrm{XV}^{\mathrm{e}}$ et $\mathrm{XVI}^{\mathrm{e}}$ siècle relatifs aux anciennes régions de Phrygie et de Bithynie, dont les registres chronologiques de biens fonciers peuvent établir l'historicité de plusieurs personnages. Même les récits hagiographiques, remplis de "contes et de fables " peuvent utilement être confrontés aux chroniques. Les travaux scientifiques tirent ainsi peu à peu la légende vers l'historicité. Et l'histoire comme la littérature y trouveront leur compte.

\section{NOTES}

1. Taner Timur, Osmanlı Çalışmaları, Imge Kitabevi, Istanbul 1998, p. 10.

2. Des auteurs comme Feridun Fazıl Tülbentçi, Turhan Tan, Abdullah Ziya Kozanoğlu, Nizamettin Nazıf, Nihal Adsız ou Mustafa Sepetçioğlu parlent de héros légendaires ou de périodes remplies de brillantes conquêtes de l'histoire ottomane ou d'Asie centrale, mais les romans produits par ce type d'écrivains entre les années 20 et 60 sont mis au ban de la littérature officielle en tant que « triviaux » ou « tendancieux ».

3. Système de Production de Type asiatique en opposition au régime féodal d'organisation des terres occidental, voir à ce sujet: Sencer Divitçioğlu, Asya Üretim Tarzı ve Osmanlı Toplumu, Marksist Üretim Tarzı Kavramı, Yapı Kredi Yayınları, Istanbul 2003.

4. Jeu de mots sur le nom de l'auteur et le mot tarih : histoire.

5. Tarık Buğra, Politika Dışı, Ötüken, Istanbul 1995, p. 214.

6. Cf. Lettres de prison de Nazım Hikmet à Kemal Tahir dans De l'espoir à vous faire pleurer de rage, Voix, François Maspero, Paris, 1973.

7. Fatih Anda, Tarık Buğra, şûle, Istanbul 1995, p. 167.

8. Tarık Buğra'dans Notlar, Ötüken Neşriyat, Istanbul, 1996, p. 236.

9. Ismet Bozdağ, « Düsunen Adam », Türkiye Defteri, Janvier 1974 p. 2.

10. Ismet Bozdağ, Kemal Tahir'in Sohbetleri, Emre Yayınları, Istanbul, 1995, p. 82.

11. Elizabeth Zachariadou, Osmanlı Beyliği (1300-1389), Tarih Vakfı Yurt Yayınları, 1997, p. 77.

12. Ibid. p. 100

13. Ismet Bozdağ, Kemal Tahir'in Sohbetleri, Emre Yayınları, Istanbul, 1995, pp. 81 et 83.

14. Devlet Ana, p. 174.

15. Paul Wittek, Osmanlı İmparatorluğu'nun Doğuşu, Pencere Yayınları, Istanbul 2000. 
16. Le terme d'ahilik véhicule une dimension spirituelle et religieuse à laquelle Kemal Tahir a préféré substituer une coloration plus pragmatique et économique. Ortak pourrait se traduire par partenariat ou marché commun.

17. Berna Moran, Türk Romanına Eleștirel Bir Bakış, İletişim Yayınları, Istanbul 2001, pp. 211-241.

18. İsmet Bozdağ, Kemal Tahir'in Sohbetleri, Emre Yayınları, Istanbul 1995, p. 82.

19. Tarık Buğra, Osmancık, p. 9.

20. Ibid. p. 38.

21. Kemal Tahir, Notes parues dans le journal Milliyet, 30 mars 1989.

22. Kemal Tahir, Devlet Ana, p. 176.

23. Murat Belge, Edebiyat Üstüne Yazılar, İletişim Yayınları, Istanbul, 1998, pp. 184-186.

24. Taner Timur, Osmanlı Türk Romanında Tarih, Toplum ve Kimlik, Imge, Ankara 2002, p. 216.

25. Ismet Bozdağ, Kemal Tahir'in Sohbetleri, Emre, Istanbul 1995, p. 83.

26. Yağmur Tunalı, “Tarık Buğra ile Osmancık Üzerine Bir Mülakat”, Töre, mars 1984, in Politika Dışı, p. 234.

27. Selim İleri, “Kemal Tahir'le Konuşma”, Yeni Dergi, Juin 1973, p.105.

28. Tarık Buğra, Bu Çağın Adı, Ötüken Neşriyat, Istanbul, 1990, pp. 404-408.

29. Tarık Buğra, dans Notlar, Ötüken, Istanbul, 1996, p. 157.

30. Toker Bilge Bayram, “Osmancık ve Tarık Buğra Anlayışı”, Erguvan, juillet 1985.

31. Tarık Buğra, Politika Dışı, Ötüken, Istanbul 1995, p. 26.

32. Kemal Kahraman, “Tarık Buğra ve Kemal Tahir'in Tarihe Bakışı”, Türk Edebiyatı, mars 1993, p. 53.

\section{RÉSUMÉS}

En traitant majoritairement du déclin de l'Empire ottoman et de l'épopée républicaine, le roman turc du début du XX $X^{\mathrm{e}}$ siècle se fait amplement l'écho de la vision officielle de l'État kémaliste moderniste et occidentaliste. Mais au fil du temps, conscients qu'il est vital pour une société de connaître son histoire, des écrivains se repenchent sur le passé ottoman.

Dans Devlet Ana (1967) et Osmancık (1983), Kemal Tahir et Tarkk Buğra prennent pour sujet le début de la fondation d'un ordre étatique par les tribus turkmènes aux XIII ${ }^{\mathrm{e}}$ et XIV ${ }^{\mathrm{e}}$ siècles. Vision marxiste pour l'un, plus spiritualiste pour l'autre, tous deux s'attachent à mettre en lumière les aspects positifs qui ont présidé à la fondation de l'Empire ottoman (défense des valeurs humaines, modèle de droit et de justice) et se rejoignent dans une même défense de l'identité nationale.

One of the prominent themes of Turkish literature of the 20th century adresses the decline of the Ottoman Empire and the emergence of the Republic. This largely echoes the official view of the western orientated Kemalistic government. Gradually, some writers became conscious of the vital necessity for the country to embrace its history, and started to examine their ottoman past. In Devlet Ana (1967) and in Osmancık (1983), Kemal Tahir and Tarık Buğra took as a subject the beginning of the foundation of a State by the tribes of Turkmen in the 13th and 14th centuries. Despite a marxist vision on one side and a spiritual view on the other, both of them were attached to putting emphasis on the positive aspects of the ottoman Empire's foundation ( the defense of human values and the model of law and order); they both came together to stand up for the defense of a national identity. 
INDEX

Index géographique: Turquie

Mots-clés : Abdal, Aga, Buğra Tarık (1918-1994), Namık Kemal (1840-1888), Ayan, Buğra Tarık (1918-1994), littérature turque, Kemal Tahir (1910-1973), identité turque, Hodja, Kayı, Turkmènes, Mollah, Namık Kemal (1840-1888), Kemal Tahir (1910-1973)

Thèmes : Littérature

Index chronologique : Empire ottoman

motsclestr Türkiye, Osmanlı İmparatorluğu

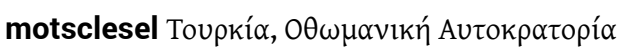

motsclesmk ОТОМАНСКАТА ИМПЕРИЈА, ТУРЦИЈА

Keywords : Buğra Tarık (1918-1994), Turkmen, Ottoman empire, Turkey, Tahir Kemal (1910-1973) 